University of Nebraska - Lincoln

DigitalCommons@University of Nebraska - Lincoln

2008

Unoccupied orbital energies of 1,4-benzenedithiol and the

HOMO-LUMO gap

Adam M. Scheer

Gordon A. Gallup

Paul Burrow

Follow this and additional works at: https://digitalcommons.unl.edu/physicsburrow

Part of the Atomic, Molecular and Optical Physics Commons

This Article is brought to you for free and open access by the Research Papers in Physics and Astronomy at DigitalCommons@University of Nebraska - Lincoln. It has been accepted for inclusion in Paul Burrow Publications by an authorized administrator of DigitalCommons@University of Nebraska - Lincoln. 


\title{
Unoccupied orbital energies of 1,4-benzenedithiol and the HOMO-LUMO gap
}

\author{
A. M. Scheer, G. A. Gallup, and P. D. Burrow \\ Department of Physics and Astronomy, University of Nebraska-Lincoln, \\ Lincoln, NE 68588-0111, United States \\ Corresponding author - P. D. Burrow, email pburrow1@unl.edu
}

\begin{abstract}
The energies of the temporary anion states of 1,4-benzenedithiol (BDT) are determined by electron scattering and compared with those computed by density functional theory (DFT) and Hartree-Fock (HF) methods. Using semi-empirical scalings derived from the ionization energies and electron affinities of a series of phenyl-ethynyl compounds, we compute the HOMO-LUMO energy gap of BDT. We show that without such scalings the gap is substantially underestimated using DFT and overestimated using HF methods. Scaled gaps are also determined for several related compounds and compared with experimental measurements in the literature.
\end{abstract}

\section{Introduction}

Spurred by the experimental work of Reed et al. [1], 1,4-benzenedithiol (BDT) bound between two gold leads has received a great deal of theoretical attention as a prototype molecular wire [2-9]. To our

Published in Chemical Physics Letters 466 (2008), pp 131-135.

doi:10.1016/j.cplett.2008.10.057

Copyright (C) 2008 Elsevier B.V. Used by permission.

Submitted 19 September 2008; revised 17 October 2008; published 1 November 2008 
knowledge, the data reported in Ref. [1] have not been experimentally confirmed by others. Theoretical studies have generally reproduced the qualitative shape of the $I-V$ curve, but have differed significantly in its magnitude and in the assignment of specific molecular states responsible for resonant electron transport.

Although the electronic states of the central molecule broaden and shift [2] upon bonding to metallic leads, accurate computed orbital energies for the unperturbed central molecule are an important reference point for more detailed calculations on the extended system. In particular, the magnitude of the HOMO-LUMO (HL) gap and its placement relative to the Fermi level of the metal leads play a significant role in device conductivity. It is not widely appreciated that DFT, now extensively employed in the modeling of molecular electronics, can result in anion state energies and HL gaps that are in considerable error. However, computed orbital energies for a given family of molecules may be correlated with their experimental counterparts, if available, to produce semiempirical scalings that can be applied to correct the computed energies in other chemically related molecules [10].

In the present work we focus on the transient occupation of the normally unoccupied orbitals of gas phase BDT observed by means of electron transmission spectroscopy (ETS) [11]. We report the energies required to inject electrons into the lowest few antibonding orbitals of BDT and discuss the role of molecular geometry on the energies, ordering and structure of these orbitals. Scalings for the filled and empty orbitals of a series of compounds containing alternating phenyl and ethynyl groups are obtained as described elsewhere [10] and used to compare with the measured energies of the transient anion states of BDT. The scalings are also used to compute the HL gap of BDT using both DFT and Hartree-Fock (HF) calculations. The results illustrate the magnitude of the errors in the uncorrected gap energies of each method.

Finally, we compute scaled HL gap energies for several compounds in their gas phase forms and compare these energies with those measured experimentally with the molecules on surfaces or in self-assembled molecular (SAM) layers. 


\section{Experiment}

Briefly, in ETS a monoenergetic electron beam is transmitted through a collision cell containing a sample gas. Scattered electrons are rejected at a retarding electrode following the collision cell and the unscattered electrons are collected. To accentuate resonant structure in the cross section, a small modulation voltage is applied directly to the collision cell and the AC component of the transmitted current is sent to a lock-in amplifier, enabling detection of the derivative of transmitted current with respect to energy [11]. In the derivative signal, a peak in the total cross section is thus signaled by a minimum followed by a maximum. The energy of a resonance is assigned by locating the vertical midpoint of this dip to peak structure. Because the attachment process is rapid relative to nuclear motion, the resonance energy characterizes the molecule in its neutral equilibrium geometry and is a measure of the vertical attachment energy (VAE).

A peak at $2.46 \mathrm{eV}$ in the derivative signal of $\mathrm{N}_{2}$ associated with the $v=2$ vibrational level of the ${ }^{2} \Pi_{\mathrm{g}}$ anion state is used to calibrate the energy scale in this work. A beam with energy width $40 \mathrm{meV}$ generated by a trochoidal electron monochromator [12] was used. The absolute uncertainty in the determination of the energy of a resonance is taken to be $\pm 50 \mathrm{meV}$. The sample has very low vapor pressure at room temperature and was introduced into the collision cell by means of a sample oven directly attached to the collision chamber. The sample was heated to $\sim 50{ }^{\circ} \mathrm{C}$ with the collision cell kept slightly warmer to avoid condensation.

\section{Results}

In Figure 1 we present the ET spectrum of BDT, plotting the derivative of transmitted current as a function of electron energy. Vertical lines mark the locations of the temporary anion states described below. The spectrum matches closely that of $p$-dimethylthiobenzene ( $p$-DTMB) presented by Modelli et al. [13]. In increasing order of energy, the four structures were assigned by these authors to the $b_{1}$ and $a_{2} \pi^{*}$ orbitals (in $C_{2 v}$ notation), derived from the degenerate $e_{2 u}$ benzene orbitals, a $\sigma^{*}$ orbital involving the sulfur atom, and to an orbital 


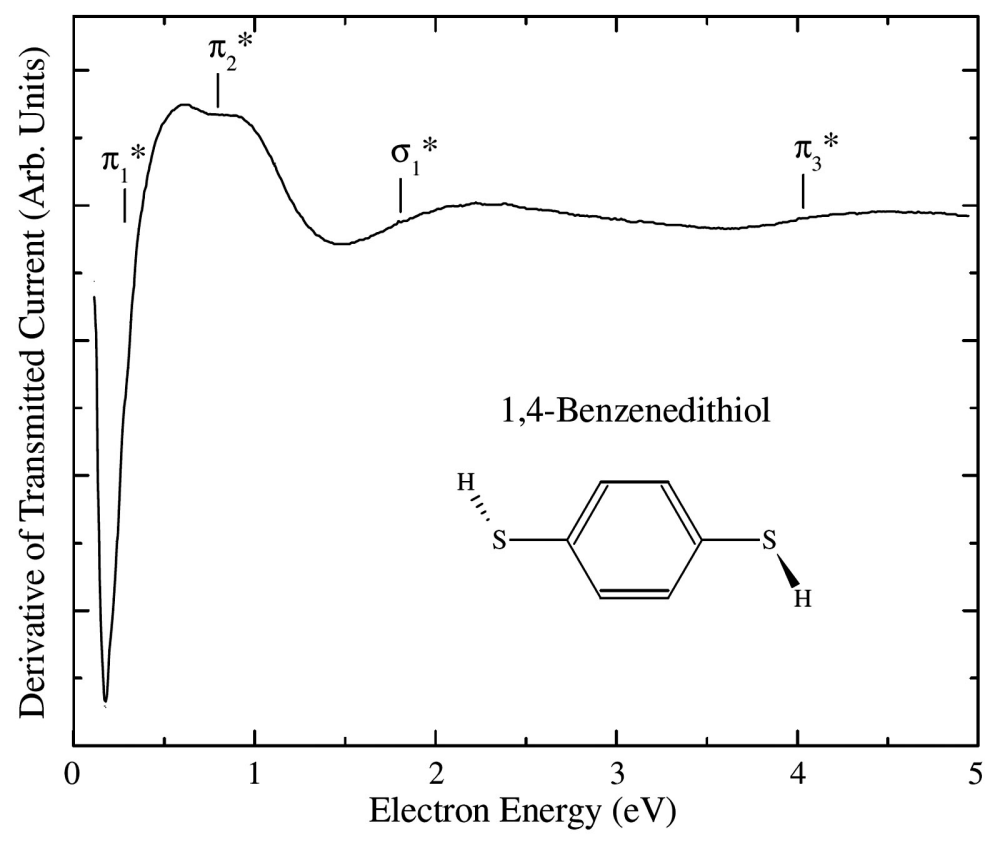

Figure 1. The derivative with respect to electron energy of current transmitted through gas phase 1,4-benzenedithiol (BDT). Vertical attachment energies (VAEs) are indicated by short vertical bars and labeled to indicate the empty orbital responsible for the resonance.

corresponding to the upper benzene $b_{2 g} \pi^{*}$ orbital. Because the ETS data do not supply direct evidence for the assignment of the two lowest anion states, split in energy by more than $500 \mathrm{meV}$, Modelli et al. inferred that $b_{1}$ was stabilized relative to $a_{2}$ from considerations of the ET spectra of mono-substituted thiobenzenes [14,15].

A key issue in the interpretation of the BDT spectrum concerns the geometry of the neutral ground state of the molecule. Similar to oxygen, sulfur retains a lone pair of electrons when bound in a molecule. Thus R-S-R bonding arrangements adopt a bent geometry. In Figure 1 we have shown the conformation with the terminal $\mathrm{H}$ atoms above and below the plane of the phenyl ring, which as we explain below is more consistent with our data than the planar molecule. Both conformers are characterized by the $\mathrm{C}_{2 \mathrm{~h}}$ point group.

As an aid in clarifying the gas phase geometry adopted by BDT and $p$-DTMB [13], geometry was optimized using the Hartree-Fock (HF) method and the 6-31G(d) basis set in the GAMESs program suite [16]. The unfilled orbitals generated by the SCF calculation are known as virtual orbitals (VOs). According to Koopmans' theorem [17], the 
energy of a VO can be used to approximate the energy of the anion resulting from occupation of that orbital. However, the computed energies of the VOs are too high with this basis set primarily because interactions with continuum states are not taken into account. Because the VOEs are close to the vacuum level in the continuum, most continuum states lie at higher energies and if included properly would push the VOEs downward in a perturbative sense. Neglect of electron correlation also induces error in the same direction.

Closer agreement with experimental VAE values may be obtained by using semi-empirical scaling procedures based on the trend between VOEs and VAEs of previously studied molecules, as discussed by Chen and Gallup [18] and Staley and Strnad [19]. A strong linear correlation between VAE and VOE was found earlier by Modelli [20] for a wide selection of unsaturated compounds with VOEs determined at the B3LYP/6-31G(d) level of theory. Similar correlations were recently demonstrated for the $\pi^{*}$ anion states of aromatic molecules consisting of alternating phenyl and ethynyl groups [10]. The relationship between measured VAEs associated with electron capture into $\pi^{*}$ orbitals of these molecules and the corresponding calculated VOEs was well described by the pair of scalings given in Eqs. (1) and (2). In Eq. (1), the VOEs were determined by HF calculations with the 6-31G(d) basis set, while those given in Eq. (2) were the result of scaling B3LYP/6-31G(d) calculations.

$$
\begin{aligned}
& \operatorname{VAE}(\mathrm{eV})=\frac{\operatorname{VOE}_{\mathrm{HF}}-2.22}{1.69} \\
& \operatorname{VAE}(\mathrm{eV})=\frac{\operatorname{VOE}_{\mathrm{DFT}}+1.14}{1.24}
\end{aligned}
$$

For our application to BDT and $p$-DTMB we will use Eq. (1) to produce scaled VOEs (SVOEs) that we identify as our theoretically predicted VAEs. Both Eq. (1) and (2) will be used later to calculate scaled HL gaps of the planar and perpendicular forms of BDT. Unfortunately, scaling procedures for $\sigma^{\star}$ orbital energies are not as well established. In this work, we apply a scaling determined for $\sigma^{\star}(\mathrm{C}-\mathrm{Cl})$ orbitals [21] to those resonances associated with $\mathrm{S}$ atom bonding. (The scaling is given by $\mathrm{VAE}=(\mathrm{VOE}-2.83) / 1.11 \mathrm{in} \mathrm{eV}$.) These energies are likely to be less accurate than those for the $\pi^{*}$ orbitals. 
Table 1 gives the VAEs of both BDT and p-DTMB [13] along with our SVOE values for each of the conformers discussed above. In both molecules the scaled LUMO energies agree well with the experimental VAEs for the conformer in which the hydrogens are bent out of plane (designated as perpendicular). The higher SVOEs also closely match their corresponding VAEs. In contrast, the scaled LUMO energies for the planar conformers of both molecules are nearly 500 meV higher than experiment and very little energy splitting is predicted between the LUMO and LUMO + 1. Thus our calculations indicate that the $b_{1}$ orbital lies lower in energy and the ET spectrum arises primarily from the non-planar form of BDT. This is consistent with previous work $[14,15]$ in which it was concluded that there should be significant splitting of the $b_{1}$ and $a_{2}$ orbitals if the thiol hydrogen atoms are bent out of the plane of the ring. As shown in Table 1, our calculations indicate that the nearly degenerate LUMO and LUMO +1 of the planar conformer of BDT are very close in energy to the LUMO +1 of the perpendicular conformer. This is not surprising because these orbitals will have little overlap with the $\mathrm{C}-\mathrm{S}-\mathrm{H} \sigma$ framework and will therefore remain close in energy to the analogous orbitals in benzene. Molecular orbital sketches for the $\pi *$ anion states of BDT in both conformers are shown in Figure $\mathbf{2}$ as generated by MOLEKEL software [22].

Table 1 Vertical attachment energies (VAEs) and scaled virtual orbital energies (SVOEs) in eV obtained from Eq. (1) for both the planar and perpendicular forms of the indicated compounds as calculated by geometry optimized Hartree-Fock quantum chemical calculations at the $6-31 \mathrm{G}(\mathrm{d})$ level. The designations in parenthesis indicate the orbital associated with that value.

\begin{tabular}{lllll} 
& $\pi_{1}{ }^{*}$ & $\pi_{2}{ }^{*}$ & $\sigma_{1}{ }^{*}$ & $\pi_{3}{ }^{*}\left(\mathrm{~b}_{2}\right)$ \\
\hline 1,4-Benzenedithiol & & & & \\
VAE & 0.29 & 0.85 & $1.84^{\mathrm{b}}$ & 4.05 \\
SVOE (perpendicular) & $0.35\left(\mathrm{~b}_{1}\right)$ & $0.77\left(\mathrm{a}_{2}\right)$ & 1.72 & 4.12 \\
SVOE (planar) & $0.76\left(\mathrm{a}_{2}\right)$ & $0.79\left(\mathrm{~b}_{1}\right)$ & 1.22 & 4.13 \\
p-Dimethylthiobenzene & & & & \\
VAE $^{\text {a }}$ & 0.39 & 0.83 & 2.3 & 4.4 \\
SVOE (perpendicular) & $0.38\left(\mathrm{~b}_{1}\right)$ & $0.83\left(\mathrm{a}_{2}\right)$ & 2.04 & 4.38 \\
SVOE (planar) & $0.87\left(\mathrm{a}_{2}\right)$ & $0.95\left(\mathrm{~b}_{1}\right)$ & 2.04 & 4.30 \\
\hline
\end{tabular}

a. VAEs obtained from Ref. [13].

b. Upper bound. 


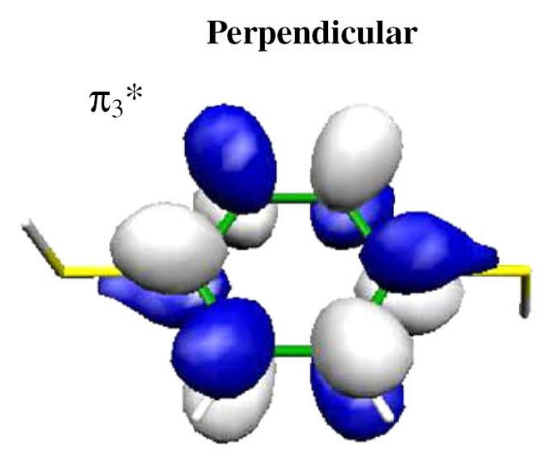

$$
\mathrm{VAE}=4.05 \mathrm{eV}
$$$$
\mathrm{SVOE}=4.12 \mathrm{eV}
$$

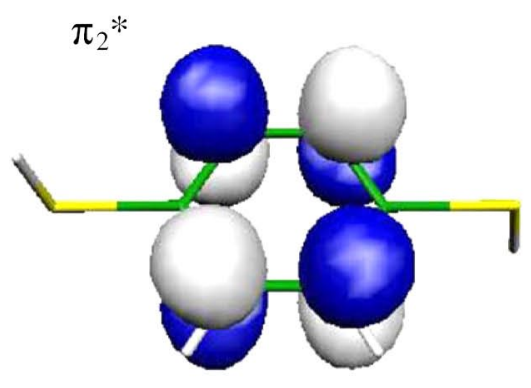

$$
\begin{aligned}
& \mathrm{VAE}=0.85 \mathrm{eV} \\
& \mathrm{SVOE}=0.77 \mathrm{eV}
\end{aligned}
$$

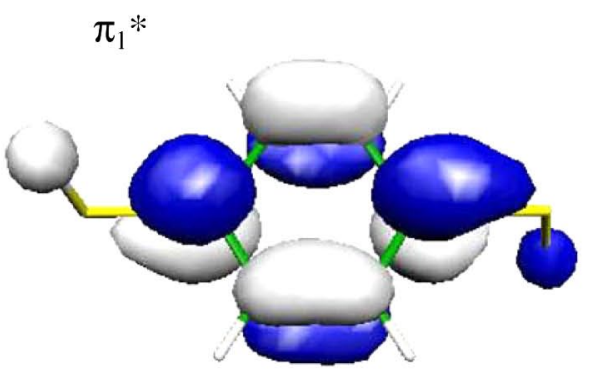

$$
\begin{aligned}
& \mathrm{VAE}=0.29 \mathrm{eV} \\
& \mathrm{SVOE}=0.35 \mathrm{eV}
\end{aligned}
$$

Planar

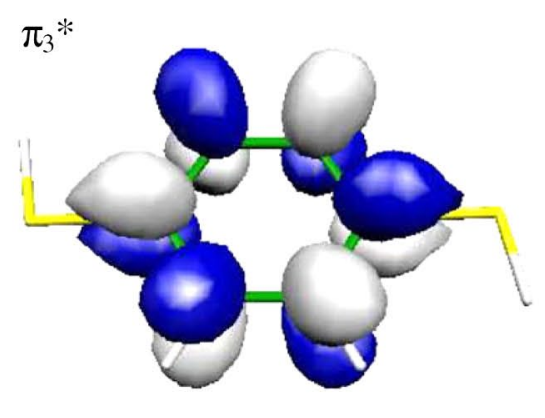

$\mathrm{SVOE}=4.13 \mathrm{eV}$

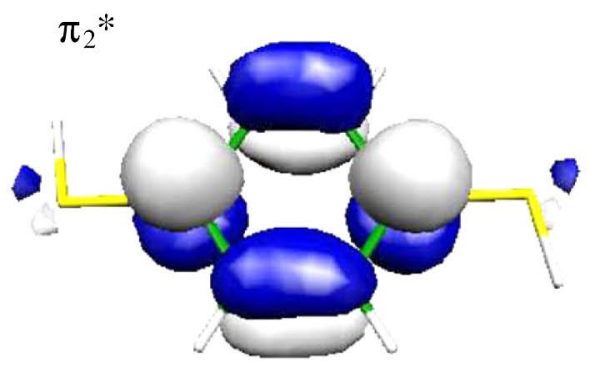

$\mathrm{SVOE}=0.79 \mathrm{eV}$

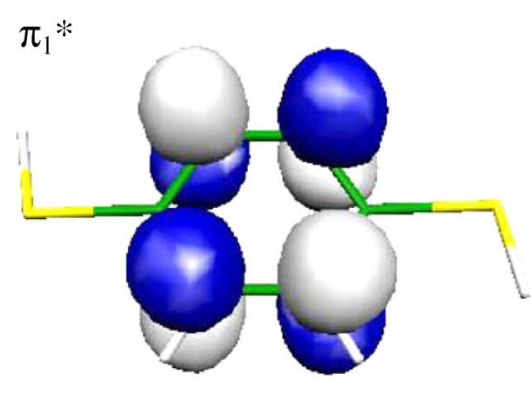

$\mathrm{SVOE}=0.76 \mathrm{eV}$

Figure 2. The normally unoccupied $\pi *$ orbitals of 1,4 -benzenedithiol (BDT) for both the planar and perpendicular conformers as calculated by geometry optimized HF calculations at the 6-31G(d) level. Vertical attachment energies (VAEs) and scaled virtual orbital energies (SVOEs) calculated with Eq. (1) are given. 
The resonance labeled $\sigma_{1}{ }^{*}$ in Figure 1 has an apparent midpoint lying at $1.84 \mathrm{eV}$. However, since $\sigma^{\star}$ resonances are expected to be rather broad, the lower part of the resonance may be obscured by the strong $\pi_{2}{ }^{*}$ feature. Thus this energy should be considered an upper bound. We also note the possibility that the profile of this resonance on its high energy side may be affected by overlap with the next higher $\sigma^{*}$ orbital. The SVOE for $\sigma_{2}{ }^{*}$ is $2.26 \mathrm{eV}$, near the peak of the derivative structure.

Modelli et al. $[14,15]$ in previous studies of monothiol benzenes have attributed a similar feature to a $\sigma^{\star}$ resonance with significant sulfur d character, localized on the S-H bond. These studies were based in part on MSXa calculations. A plot of the lowest unoccupied $\sigma^{*}$ orbital of BDT is shown in Figure 3. Our scaled HF calculations locate the energies of pure $\sigma_{3,4}{ }^{*} \mathrm{~S}-\mathrm{H}$ orbitals at $2.5 \mathrm{eV}$ and higher, whereas the SVOE corresponding to the $\sigma_{1}{ }^{*}$ orbital is $1.72 \mathrm{eV}$, in good agreement with experiment. An examination of the atomic orbital coefficients of this orbital indicates there is negligible sulfur $d$ involvement and that the primary contributors are actually sulfur $3 p$ orbitals. Calculations were also carried out with and without the $\mathrm{S} 3 \mathrm{~d}$ orbitals included in the basis set. SVOEs are given in Table $\mathbf{2}$ for both the planar and perpendicular forms of BDT. Other than a slight stabilization of the $\mathrm{a}_{2}$ orbital of the planar form of BDT, our scaled HF/6-31G(d) calculations show very little difference in the orbital energies when sulfur $3 \mathrm{~d}$ functions are removed.

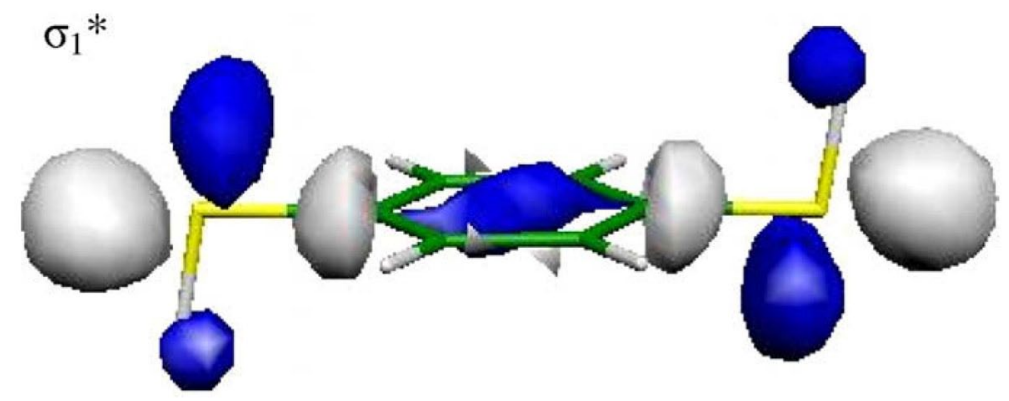

Figure 3. The lowest unoccupied $\sigma^{*}$ orbital in 1,4-benzenedithiol (BDT) as calculated by geometry optimized HF calculations at the 6-31G(d) level. 
Table 2 Scaled virtual orbital energies in eV resulting from geometry optimized HF calculations for the planar and perpendicular forms of 1,4-benzenedithiol (BDT) with and without $S 3$ d orbitals included in the $6-31 \mathrm{G}(\mathrm{d})$ basis set.

\begin{tabular}{lllll} 
SVOEs $(\mathrm{eV})$ & $\pi_{1}{ }^{*}$ & $\pi_{2}{ }^{*}$ & $\sigma^{*}$ & $\pi_{1}{ }^{*}$ \\
\hline BDT perpendicular & & & & \\
With S 3d orbitals & 0.35 & 0.76 & 1.72 & 4.12 \\
Without S 3d orbitals & 0.37 & 0.70 & 1.69 & 4.20 \\
BDT planar & & & & \\
With S 3d orbitals & 0.76 & 0.79 & 1.73 & 4.13 \\
Without S 3d orbitals & 0.68 & 0.79 & 1.67 & 4.21 \\
\hline
\end{tabular}

\section{Scaled HOMO-LUMO gap of BDT}

The HOMO-LUMO (HL) gap of a molecular wire will play an important role in determining its conductance properties [23,24]. In HF calculations, the energy of the HOMO approximates -IE, the negative of the ionization energy according to Koopmans' theorem. Similarly, the negative of the energy of the LUMO is identified with $\mathrm{EA}_{\mathrm{v}}$, the vertical electron affinity, that is, the electron affinity of the molecule in its neutral ground state geometry. The HL gap is thus given experimentally by IE - EA $\mathrm{v}_{\mathrm{v}}$. While the HOMO energy is similarly identified with -IE in DFT calculations, the LUMO energy is generally regarded as a poor representation for $-\mathrm{EA}_{\mathrm{v}}[25]$, and the HL gap is shown to be an approximation of the lowest excitation energy of an individual molecule [26] or of the band gap in crystalline solids. In the application of DFT to molecules, the calculated HL gap may therefore differ substantially from IE - $\mathrm{EA}_{\mathrm{v}}$. In contrast to the IE, which can be readily measured with photoelectron spectroscopy, it is worth noting that a positive $\mathrm{EA}_{\mathrm{v}}$ is not readily accessible experimentally in the gas phase. Negative $\mathrm{EA}_{\mathrm{v}} \mathrm{s}$, on the other hand, can be determined experimentally by observing resonances in electron scattering as shown here. We note that $\mathrm{VAE}=-\mathrm{EA}_{\mathrm{v}}$.

As alluded to earlier, both the HF and DFT LUMOs can be empirically scaled to match experimental EAs. Similarly, the occupied orbital energies (OOEs), though initially more accurate, can be scaled to the IEs. Eqs. (3) and (4) show the scaling for the latter based on data in the phenylacetylenes [10] for both methods of calculation. 


$$
\begin{aligned}
& \mathrm{IE}(\mathrm{eV})=\frac{-\mathrm{OOE}_{\mathrm{HF}}+2.88}{1.30} \\
& \mathrm{IE}(\mathrm{eV})=\frac{-\mathrm{OOE} E_{\mathrm{DFT}}+1.21}{0.85}
\end{aligned}
$$

In Table 3 we present the scaled and unscaled HOMO and LUMO energies of BDT in the planar and perpendicular geometries using both HF and DFT calculations. The scaled HL gaps obtained by both methods agree to within $0.42 \mathrm{eV}$ for a given conformer. As discussed in Ref. [10], in the absence of scaling, HL gaps computed using DFT are substantially underestimated. In contrast, HF methods yield a sizeable overestimate of the HL gap primarily because of the error in the LUMO energy.

\section{Illustrations of scaled HOMO-LUMO gaps}

We have shown that the measured temporary $\pi^{*}$ anion state energies of BDT are in good agreement with the scaled orbital energies obtained from either DFT or HF calculations. The scalings employed here are obtained from data in a set of phenyl-ethynyl compounds studied earlier [10]. We do not propose that these scalings are universal and emphasize that in general such scales should be obtained from other compounds as closely related to the molecule under study as possible.

Table 3 HOMO and LUMO energies calculated at the HF/6-31G(d) and DFT/631G(d)/B3LYP levels and scaled energies resulting from application of Eqs. (1)-(4) for the compounds indicated. The HOMO-LUMO (HL) gaps are also given. All energies are in $\mathrm{eV}$.

\begin{tabular}{lrrrrrr} 
Compound & HOMO LUMO & HL gap & $\begin{array}{r}\text { Sc.HOMO } \\
\text { (Eq. (3)) }\end{array}$ & $\begin{array}{r}\text { Sc.LUMO } \\
\text { (Eq. (1)) }\end{array}$ & $\begin{array}{r}\text { Sc.HL } \\
\text { Gap }\end{array}$ \\
\hline $\begin{array}{l}H F / 6-31 G(d) \\
\quad\end{array}$ & & & & & & \\
BDT planar & -7.98 & 3.5 & 11.48 & -8.35 & 0.76 & 9.11 \\
$\begin{array}{l}\text { BDT perpendicular } \\
\text { DFT/6-31G(d)/B3LYP }\end{array}$ & -9.48 & 2.81 & 12.29 & -9.51 & 0.35 & 9.86 \\
BDT planar & -5.55 & -0.22 & 5.33 & -7.95 & 0.74 & 8.69 \\
BDT perpendicular & -6.68 & -0.83 & 5.85 & -9.28 & 0.25 & 9.53 \\
\hline
\end{tabular}


However, we expect that the scalings presented in Ref. [10] are appropriate for many of the long chain aromatics commonly used in molecular electronics and various SAM studies.

To explore these considerations and investigate the effect of the metal surface on HL gaps, we comment briefly on a few examples of published research dealing with SAMs in which HL gaps are both computed and measured. In a study of biphenyldimethyldithiol ( $\mathrm{HS}-\mathrm{CH}_{2}-$ $\mathrm{C}_{6} \mathrm{H}_{4}-\mathrm{C}_{6} \mathrm{H}_{4}-\mathrm{CH}_{2}-\mathrm{SH}$ ) deposited from solution on a $\mathrm{Au}(111)$ surface [27], the occupied and unoccupied electronic structure was explored using angle resolved photoemission (PES) and inverse photoemission spectroscopy (IPES), respectively. According to the authors, the deposition process led to well ordered molecular films about five or more monolayers thick. The experimental data are accompanied by HF calculations of the filled and empty orbital energies using the $6-31 \mathrm{G}(\mathrm{d})$ basis set. These lead to a 'theoretical' HL gap of $10.33 \mathrm{eV}$, substantially larger than the $7.8 \mathrm{eV}$ gap observed experimentally. However, using the scaling given here for HOMO and LUMO energies, the HL gap is predicted to be $8.5 \mathrm{eV}$, much closer to experiment.

As shown in Figure 2 of Ref. [27], the experimental gap of $7.8 \mathrm{eV}$ arises from placement of the HOMO energy midway up the rising PES signal rather than at the peak of this feature (which is assigned to HOMO-2). This choice is not discussed in the text but was inferred from deconvolution and an estimate of instrumental line widths (P.A. Dowben, private communication). On the other hand, the gap between the first observable peaks on each side of the Fermi level is approximately $8.6 \mathrm{eV}$. If this value is a more appropriate assignment of the HL gap, it is essentially identical to the free molecular value given by our scaling and implies that the PES and IPES signals are derived from molecules that have very little interaction with the Au surface or each other.

In a closely related study [28] of biphenyldiisocyanide carried out by the same group, the HL gap computed with HF/6-31G(d) is given as $9.98 \mathrm{eV}$ for the planar conformation of the molecule. Using the HF scaling described earlier, the HL gap is reduced to $8.3 \mathrm{eV}$. Surprisingly, the experimental gap, again determined using PES and IPES, is given as $10.8 \mathrm{eV}$, larger than the scaled gap by $2.5 \mathrm{eV}$. However, there is uncertainty in the experimental value (P.A. Dowben, private communication) because the IPES data are without sharp structure that firmly 
point to the LUMO energy. Even with a possible error reducing the experimental value by $0.5^{-1} \mathrm{eV}$, it appears that the measured gap would exceed that of the unperturbed molecule by approximately $1.5 \mathrm{eV}$.

Aside from uncertainty in the determination of the experimental HL gap, another possibility is that the planar conformer is not the correct one in the deposited film. We carried out HF calculations for the conformer in which the two phenyl rings are at right angles to each other and find a scaled HL gap of $9.7 \mathrm{eV}$, which is closer to the quoted experimental value. However, this geometry appears to be incompatible with other results described in the work (P.A. Dowben, private communication).

It is worth noting that in a more recent study of a self-assembled molecular layer of $\left[1,1^{\prime} ; 4^{\prime}, 1^{\prime \prime}\right.$-terphenyl $]-4,4^{\prime}$-dimethanethiol by the Dowben group [29], the HL gap for the gas phase molecule was computed using a semi-empirical approach, PM3-NDDO, resulting in a gap of $7.7 \mathrm{eV}$. To compare, we computed the gap using both HF and DFT methods with the 6-31G(d) basis set. With the appropriate scalings discussed earlier, we find values of 7.9 and $7.8 \mathrm{eV}$, respectively, in very good agreement, although the PM3 orbital energies themselves, however, are much too positive. We note further that the HL gap determined by DFT with no scaling is $4.2 \mathrm{eV}$, substantially smaller. Thus while reviewers frequently object to the use of semi-empirical methods, as opposed to the implied 'state of the art' DFT, it is important to recognize the failings of the latter when applied directly to single molecules.

Watkins et al. [30] have studied a monolayer of 4,40-bis(phenylethynyl) benzenethiol on Au using PES and IPES. DFT calculations using the B3LYP functional and the $6-31 \mathrm{G}(\mathrm{d})$ basis set were reported for the isolated molecule. The computed frontier orbital gap was 'contracted' by approximately $1 \mathrm{eV}$ to match the experimental HL gap of $4.7 \mathrm{eV}$. We assume therefore that the computed gap was $5.7 \mathrm{eV}$. However, our scaled DFT calculation for this compound yields a much larger HL gap of $7.2 \mathrm{eV}$ for the planar conformer and $7.3 \mathrm{eV}$ for the conformer with $\mathrm{H}$ out of plane. This comparison shows that the actual interaction with the surface arising from polarization and chemical bonding of the $\mathrm{S}$ atom to the surface causes a more substantial change of 2.5-2.6 eV rather than $1 \mathrm{eV}$.

In conclusion, we have shown that computation of HL gaps can be carried out with either HF or DFT methods, and provided that the 
appropriate scalings are used, the agreement is good. Comparison of these gas phase values with measured gaps obtained from surface experiments appears to give additional insight regarding the interactions with the surface. Given the increased usage of DFT in molecular electronics, the necessity of scaling, particularly for the empty orbitals, is stressed.

Acknowledgment We are indebted to Prof. Peter Dowben for many useful discussions.

\section{References}

[1] M.A. Reed, C. Zhou, C.J. Mueller, T.P. Burgin, J.M. Tour, Science 278 (1997) 252.

[2] A. Nitzan, M.A. Ratner, Science 300 (2003) 1384.

[3] S. Piccinin, A. Selloni, S. Scandolo, R. Car, G. Scoles, J. Chem. Phys. 119 (2003) 6729.

[4] Y. Xue, S. Datta, M.A. Ratner, J. Chem. Phys. 115 (2001) 4292.

[5] A. Kopf, P. Saalfrank, Chem. Phys. Lett. 386 (2004) 17.

[6] M. Di. Ventra, S.T. Pantelides, N.D. Lang, Phys. Rev. Lett. 84 (2000) 979.

[7] F.F. Fan et al., J. Am. Chem. Soc. 126 (2004) 2568.

[8] Y. Xue, M.A. Ratner, Phys. Rev. B 68 (2003) 115406.

[9] E. Emberly, G. Kirczenow, Nanotechnology 10 (1999) 285.

[10] A.M. Scheer, P.D. Burrow, J. Phys. Chem. B 110 (2006) 17751.

[11] L. Sanche, G.J. Schulz, Phys. Rev. A 5 (1972) 1672.

[12] A. Stamatovic, G.J. Schulz, Rev. Sci. Instrum. 41 (1970) 423.

[13] A. Modelli, D. Distefano, M. Guerra, D. Jones, S. Rossini, Chem. Phys. Lett. 132 (1986) 448.

[14] A. Modelli, D. Jones, P. Colonna, G. Distefano, Chem. Phys. 77 (1983) 153.

[15] M. Guerra, G. Distefano, D. Jones, P. Colonna, A. Modelli, Chem. Phys. 91 (1984) 383.

[16] M.W. Schmidt et al., J. Comput. Chem. 14 (1993) 1347.

[17] T. Koopmans, Physica 1 (1933) 105.

[18] D. Chen, G.A. Gallup, J. Chem. Phys. 93 (1990) 12.

[19] S.W. Staley, J.T. Strnad, J. Phys. Chem. 98 (1994) 116.

[20] A. Modelli, Phys. Chem. Chem. Phys. 5 (2003) 2923.

[21] K. Aflatooni, G.A. Gallup, P.D. Burrow, J. Phys. Chem. A 104 (2000) 7359.

[22] S. Portmann, CHIMIA 54 (2000) 766. see Molekel < http://www.cscs.ch/ molekel/ $>$.

[23] J.G. Kushmerick et al., J. Am. Chem. Soc. 124 (2002) 10654. 
[24] C. Majumder, T. Briere, H. Mizuseki, Y. Kawazoe, J. Phys. Chem. A 106 (2002) 7911.

[25] See, for example G. Zhang, C.B. Musgrave, J. Phys. Chem. A 111 (2007) 1554.

[26] Andreas Goerling, Phys. Rev. A 54 (1996) 3912.

[27] A.N. Caruso, R. Rajesh, G. Gallup, J. Redepenning, P.A. Dowben, J. Phys.: Condens. Matter. 16 (2004) 845.

[28] A.N. Caruso, R. Rajesh, G. Gallup, J. Redepenning, P.A. Dowben, J. Phys. Chem. B 108 (2004) 6910.

[29] D.-Q. Feng, D. Wisbey, Ya.B. Losovyj, Y.TaiM. Zharnikov, P.A. Dowben, Phys. Rev. B 74 (2006) 165425.

[30] N.J. Watkins et al., Chem. Phys. Lett. 446 (2007) 359. 